\title{
Magnetically inspired deformation of the liquid/vapor interface drives soap bubbles
}

Irina Legchenkova PhD

Chemical Engineering, Biotechnology and Materials Department,

Engineering Faculty, Ariel University, Ariel, Israel

Gilad Chaniel PhD

Physics Department, Exact Sciences Faculty, Ariel University, Ariel, Israel

Mark Frenkel PhD

Chemical Engineering, Biotechnology and Materials Department,

Engineering Faculty, Ariel University, Ariel, Israel

\author{
Yelena Bormashenko \\ Chemical Engineering, Biotechnology and Materials Department, \\ Engineering Faculty, Ariel University, Ariel, Israel \\ Shraga Shoval \\ Industrial Engineering and Management, Engineering Faculty, Ariel \\ University, Ariel, Israel \\ Edward Bormashenko \\ Chemical Engineering, Biotechnology and Materials Department, \\ Engineering Faculty, Ariel University, Ariel, Israel (corresponding author: \\ edward@ariel.ac.il)
}

The possibility of driving millimetrically scaled, soap and glycerol bubbles floating on a liquid, using a steady magnetic field of c. $0.5 \mathrm{~T}$, is demonstrated. The bubbles are repelled by the magnet, starting from a threshold initial lateral distance of $L_{0}^{\mathrm{cr}}=4 \mathrm{~mm}$ from the vertical axis of the magnet, and are attracted to the magnet when the initial lateral distance $L_{0}<L_{0}^{\mathrm{cr}}$. The displacement of bubbles is due to the deformation of the liquid/vapor interface by the magnetic field, known as the Moses effect. This deformation gives rise to the change in apparent contact angles driving the bubble. Switching of repulsion to attraction is explained in terms of a model of an interaction between two capillary charges, one of which represents the bubble and the second represents the well, produced by the magnet on the liquid/vapor interface. The experiments with bubbles demonstrate that the role of gravity in the displacement of floating diamagnetic objects driven by steady magnetic fields may be negligible. The mathematical model of the process is suggested. The experimental data are well explained by the proposed model.

\section{Notation}

$B_{Z} \quad$ vertical component of the magnetic field

$F_{\mathrm{dr}} \quad$ driving force

$F_{\text {visc }}$ viscous force

$h \quad$ separation of the magnet from the liquid surface

$L_{0} \quad$ initial lateral distance

$L_{0}^{\mathrm{cr}} \quad$ threshold initial lateral distance

$l \quad$ perimeter of the triple line

$R \quad$ radius of the triple line

$r \quad$ coordinate of the radial distribution of the magnetic field

$r_{\mathrm{cm}} \quad$ lateral distance between the center mass of the bubble and the axis $z$ of the magnet

$t$ time

$v_{\mathrm{cm}} \quad$ velocity of the center mass of the bubble

$\gamma \quad$ surface tension

$\Delta \theta \quad$ contact angle hysteresis

$\eta \quad$ dynamic viscosity

$\theta \quad$ interfacial angle

$\widetilde{\theta} \quad$ maximal contact angle hysteresis

$\lambda \quad$ characteristic length, describing the spatial attenuation of $B_{Z}$

$\widehat{\rho} \quad$ specific resistivity

\section{Introduction}

Applying electrical and magnetic fields at interfaces gives rise to numerous physicochemical events, including the magnetic actuation of foams, ${ }^{1}$ self-assembly ${ }^{2-6}$ and magnetocapillary valving. ${ }^{7}$ Magnetocapillary effects are usually obtained by the introduction of nano- or microferromagnetic particles to the interfaces. ${ }^{1-6}$ However, the authors demonstrated recently ${ }^{8,9}$ that floating diamagnetic objects (polymer rafts) could be directly actuated with moderate magnetic fields $(c .0 \cdot 1 \mathrm{~T})$. The authors related the effect to the driving force arising from the difference of the contact angle on both sides of the drop. This force in turn results ${ }^{10-16}$ from the deformation of the water/vapor interface by the magnetic field. ${ }^{8,9}$ The effect of deformation of liquid interfaces by magnetic fields is also called the Moses effect. ${ }^{17-19}$ However, the role of gravity (buoyancy) remained obscure. Indeed, the floating body may be displaced by the driving force arising from the difference of the contact angle on both sides of the drop, as suggested by den Dulk et al., ${ }^{7}$ and also by gravity diminishing the potential of a body in the course of its motion.

The present paper sheds light on the role of gravity in the displacement of floating diamagnetic objects driven by steady magnetic fields. The authors studied the motion of floating soap bubbles driven by a magnetic field of $c .0 .5 \mathrm{~T}$ (at the surface of the magnet $(h=0), B_{z 0}=0.52 \pm 0.01 \mathrm{~T}$; for $h=1 \mathrm{~mm}, B_{z}=$ $0.33 \pm 0.01 \mathrm{~T}$; for $h=2 \mathrm{~mm}, B_{z}=0 \cdot 26 \pm 0.01 \mathrm{~T}$; and for $h=$ $3 \mathrm{~mm}, B_{z}=0 \cdot 18 \pm 0 \cdot 01 \mathrm{~T}$ ). The radial distribution of the magnetic field is well fitted by the exponential approximation - that is, $B_{z}(r)=B_{z 0} \exp (-r / \lambda)$, with $\lambda=2 \cdot 5 \pm 0 \cdot 2 \mathrm{~mm}$. The suggested exponential decrease law of the magnetic field is justified by the fact that the stack of magnets used has a rather complicated internal structure. ${ }^{9}$ 


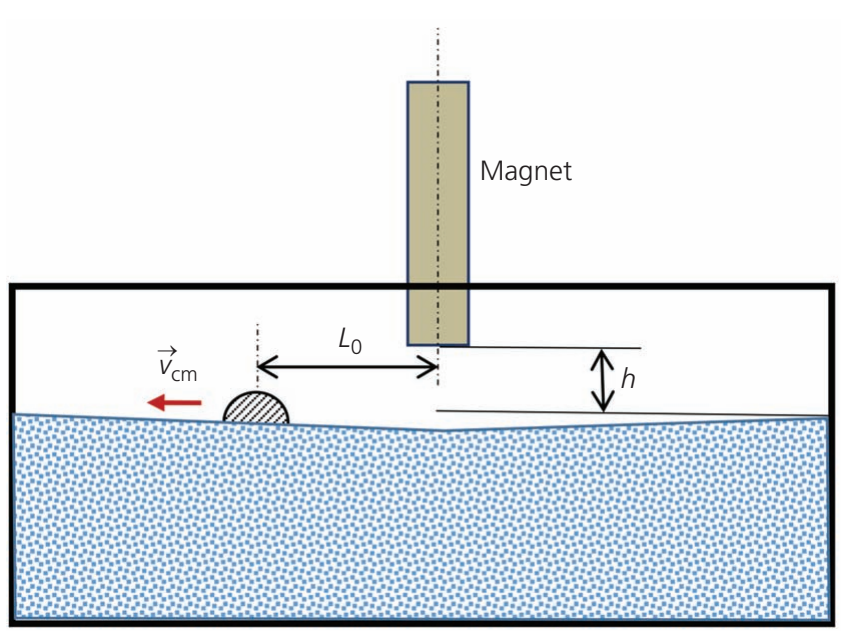

Figure 1. Scheme of the experimental unit used for the study of magnetically inspired displacement of bubbles is depicted

\section{Experimental}

The scheme of the experiment is depicted in Figure 1. The distance between the magnet and the water/vapor interface was $h=1-3 \mathrm{~mm}$. Two kinds of bubbles were investigated, namely, glycerol and soap bubbles. The glycerol dynamic viscosity $\eta$ was $(934 \pm 1) \times$ $10^{-3} \mathrm{~Pa} \mathrm{~s}$, and the surface tension $\gamma$ was $(72 \cdot 0 \pm 0 \cdot 5) \times 10^{-3} \mathrm{~N} / \mathrm{m}^{20}$

The soap bubbles were prepared as follows: air bubbles with a diameter of about $3 \pm 0 \cdot 2 \mathrm{~mm}$ and a volume of $15 \mu \mathrm{l}$ were inflated with a precise microsyringe on a $20 \%$ surfactant aqueous solution surface, placed in a petri dish with a diameter of $195 \mathrm{~mm}$. The surfactant used in the experiments was prepared as follows: $30 \mathrm{~cm}^{3}$ of oleic acid was mixed with $50 \mathrm{~cm}^{3}$ of distilled water; $73 \mathrm{~cm}^{3}$ of a $10 \%$ solution of triethanolamine, $164 \mathrm{~cm}^{3}$ of pure glycerin and $47 \mathrm{~cm}^{3}$ of distilled water were added to the mixture. ${ }^{21}$ All of the chemicals were supplied by Sigma-Aldrich.
The deionized water was prepared by using a Synergy ultraviolet water purification system from Millipore Sas (France), and its specific resistivity was $\widehat{\rho}=18 \cdot 2 \mathrm{M} \Omega \mathrm{cm}$ at $25^{\circ} \mathrm{C}$. The dynamic viscosity $\eta$ and the surface tension $\gamma$ of the solution were established as $\eta=1.45 \pm 0.01 \times 10^{-3} \mathrm{~Pa}$ s and $\gamma=26.2 \pm 0 \cdot 1 \times$ $10^{-3} \mathrm{~N} / \mathrm{m}$. The surface tension of the solution was established with the pendant drop method using a ramé-Hart 500 goniometer. The viscosity was measured by using an Ostwald viscometer.

Bubbles, such as those shown in Figure 2, were blown with the precise microsyringe at various initial lateral distances $L_{0}=$ 4-9 $\mathrm{mm}$ taken from the axis of the magnet to the axis of a bubble (see Figure 1). The precise location of the permanent magnet was fixed with a laboratory-built accurate $X Y Z$ actuator adjusted from components supplied by CCM Automation Technology. The controller used in the experiments was an Arduino controller for step motors. The motion of the soap bubbles was registered from above with a rapid camera (Nikon 1 V3). The experiments were carried out under ambient conditions.

\section{Results and discussion}

Now consider the experimental observations obtained with glycerol bubbles. Glycerol bubbles were 'repelled' from the magnet (see Video 1 in the online supplementary material) when $L_{0}>L_{0}^{\text {cr }}=$ $4 \mathrm{~mm}$ took place (where $L_{0}^{\mathrm{cr}}$ is the 'critical' initial lateral distance) and attracted by the magnet for $L_{0}<L_{0}^{\text {cr }}$ (see Video 2 in the online supplementary material). Soap bubbles were repelled from the magnet when $L_{0}>L_{0}^{\mathrm{cr}}$. It was technically impossible to place soap bubbles at smaller distances from the magnet, due to their relatively large hemispherical 'cap', which was already touching the rim of the magnet. The typical time dependencies of the velocity of the center of mass $v_{\mathrm{cm}}$ of the soap bubbles, repelled by the magnet, are presented in Figures 3(a) and 4.

The very question is, what is the physical mechanism of the repulsion of bubbles by the magnet? Consider first switching of the

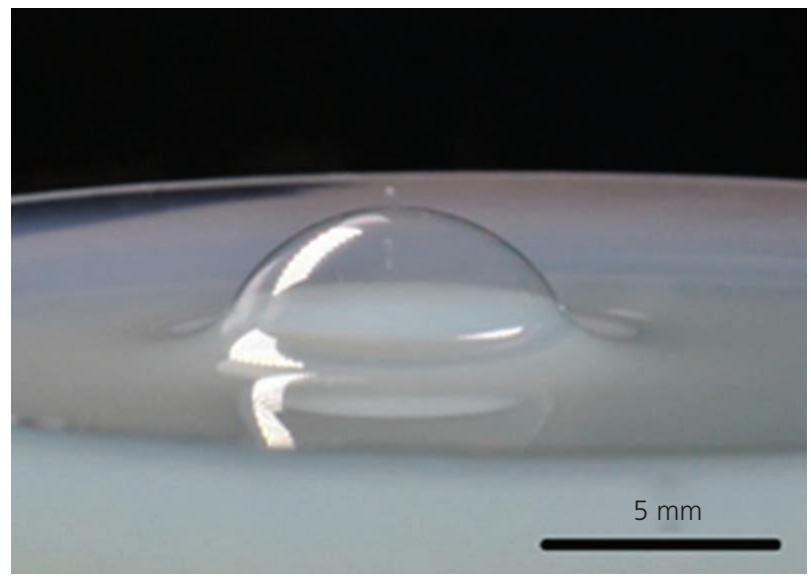

(a)

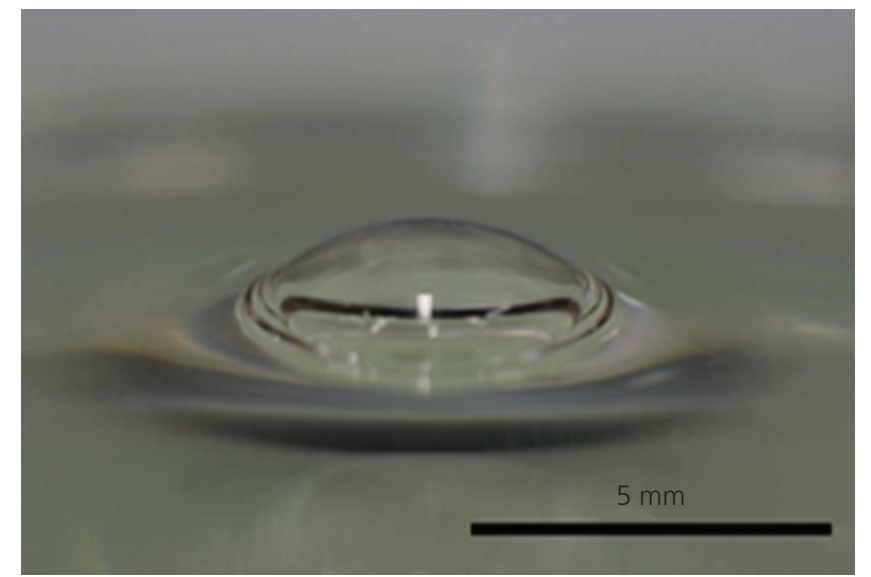

(b)

Figure 2. (a) Floating soap and (b) glycerol bubbles are shown 


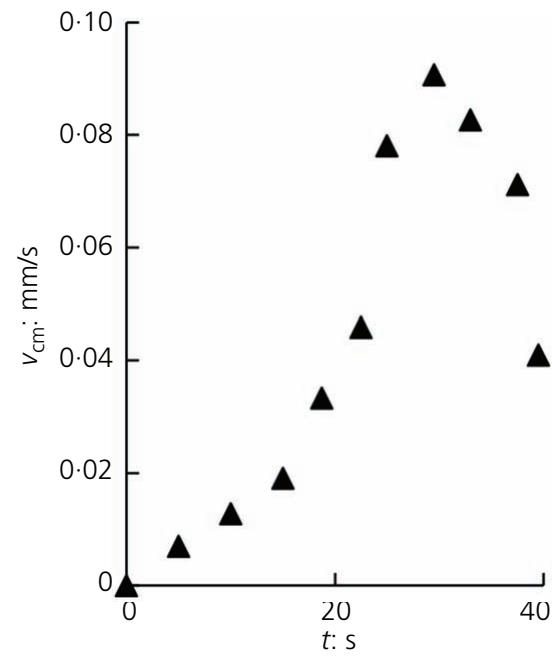

(a)

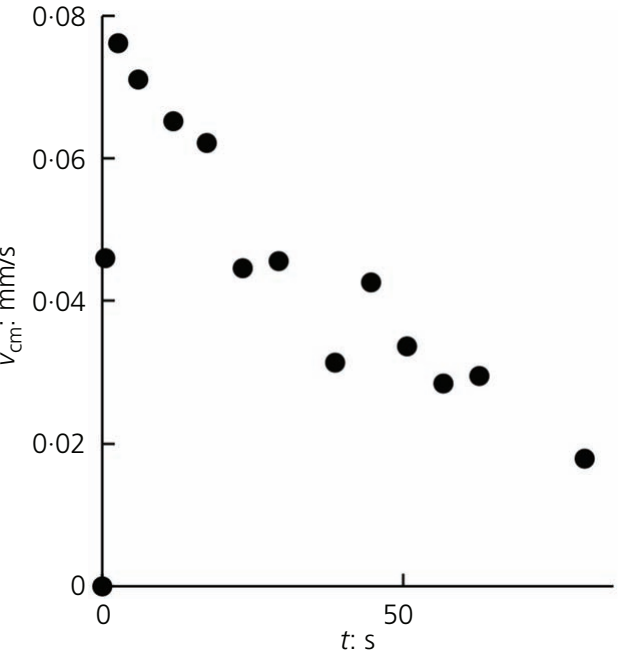

(b)

Figure 3. Time dependencies of the velocity of the center of mass $v_{\mathrm{cm}}$ of glycerol bubbles (a) attracted and (b) repelled by the magnet are presented

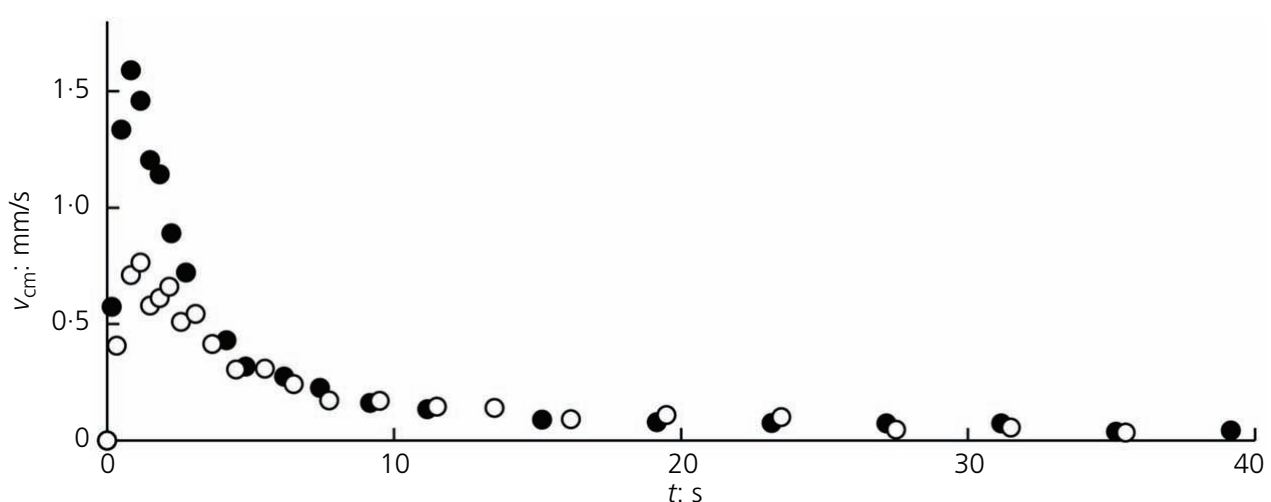

(a)

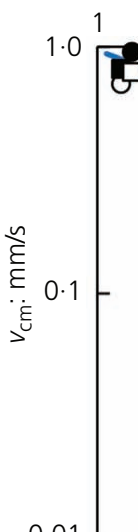

$\ln \left(t / t_{0}\right)$

10
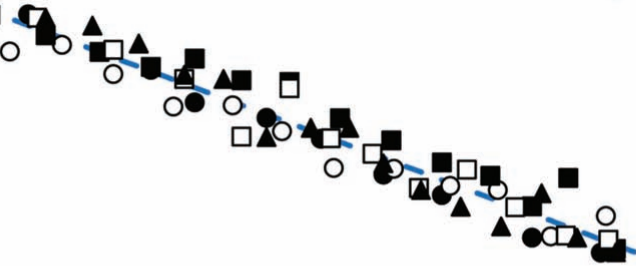

0.01

(b)

Figure 4. Time dependencies of the velocity of the center of mass $v_{\mathrm{cm}}$ of soap bubbles repelled by the magnet are depicted, where $v_{\mathrm{cm} 0}=$ $0.93 \mathrm{~mm} / \mathrm{s}$ is the averaged maximal velocity of the center mass of a bubble; $t_{0}=2 \mathrm{~s}$ is the averaged time, necessary for attaining the velocity of $v_{\mathrm{cmo}}$. (a) The experimental results obtained for various separations of the magnet from the liquid surface $h(\bullet, h=1 \mathrm{~mm} ; 0, h=$ $2 \mathrm{~mm}$ ) are presented. The diameter $d$ of the bubble was $3.3 \mathrm{~mm}$. (b) The double-logarithmic plots of the time dependence of the velocity of the center of mass of the bubble are depicted. The dashed line depicts the linear fitting of the experimental data. The water-magnet separation and the diameter of the bubble were,$h=1 \pm 0.1 \mathrm{~mm}$ and $d=3.3 \pm 0.1 \mathrm{~mm} ; 0, h=2 \pm 0.1 \mathrm{~mm}$ and $d=3.3 \pm 0.1 \mathrm{~mm}$; $h=3 \pm 0.1 \mathrm{~mm}$ and $d=3.5 \pm 0.1 \mathrm{~mm} ; \square, h=1 \pm 0.1 \mathrm{~mm}$ and $d=4 \pm 0.1 \mathrm{~mm}$; and $\boldsymbol{\Lambda}, h=2 \pm 0.1 \mathrm{~mm}$ and $d=4 \pm 0.1 \mathrm{~mm}$ 
apparent repulsion of bubbles to their attraction to the magnet observed at $L_{0}^{\text {cr }}$. This effect was already considered by Frenkel et al., ${ }^{9}$ and it is illustrated in Figure 5. The change in the apparent interaction of bubbles with the magnet is well explained by the model proposed by Kralchevsky and Nagayama ${ }^{22,23}$ and Kralchevsky et al. ${ }^{24}$ introducing the notion of the capillary charge $Q_{i}=R_{i} \sin \theta_{i}$, where $R$ and $\theta$ are the radius of the triple line and the interfacial angle of a floating bubble (curved surface), respectively. ${ }^{22-25}$ The interaction of a bubble with a magnet is seen in this model as an interaction between two capillary charges, one of which represents the bubble and the second represents the well (punctum) produced by the magnet on the liquid/vapor interface. As shown by Kralchevsky and Nagayama ${ }^{22,23}$ and Kralchevsky et al. ${ }^{24}$ the interaction is attractive when $\sin \theta_{1} \sin \theta_{2}>0$ and repulsive when $\sin \theta_{1} \sin \theta_{2}<0$, where $\theta_{1}$ and $\theta_{2}$ are the interfacial angles $^{25}$ of the well and the bubble, respectively (consider for bubbles $\sin \theta_{1}<0$ ). When a bubble is located initially at the left from the liquid surface inflection point $\mathrm{K}, \sin \theta_{2}<0$ takes place, and when it is placed at the right from the inflection point, $\sin \theta_{2}>$ 0 occurs (see Figure 5). Thus, switching of repulsion to attraction is explained. The qualitative analysis of the attraction of bubbles by the magnet is extremely challenging due to the proximity of characteristic lateral dimensions of a bubble and the well arising from the Moses effect (both of them are c. 5-10 mm).

Consider now the repulsion of pure glycerol and soap bubbles (in other words, the condition $L_{0}>L_{0}^{\text {cr }}$ takes place). Note that bubbles present an extremely convenient object for clarifying this mechanism due to the fact that all of the bulk forces (namely, buoyancy, inertia force and magnetic force ${ }^{26,27}$ ) are negligible (in other words, a bubble serves as a probe elucidating the role of interfacial effects, driving floating diamagnetic bodies ${ }^{8,9,22-25,28}$ ). Thus, the displacement of the floating bubble by the steady magnetic field is a pure interfacial effect inspired by the deformation of the liquid/vapor interface by the magnetic field. ${ }^{8,9}$

The magnetic field deforms not only the liquid/vapor interface due to the Moses effect but also the shape of the bubble, as shown in Figure 6. This deformation, which is actually possible due to the contact angle hysteresis $\left(\Delta \theta=\theta_{1}-\theta_{2}\right.$; see the papers of Chen et $a l .{ }^{16}$ and Sugawara $e t a l .{ }^{17}$ ), gives rise to the driving force, which may be roughly estimated according to the Furmidge equation ${ }^{29}$

1. $F_{\mathrm{dr}} \cong \gamma l\left(\cos \theta_{2}-\cos \theta_{1}\right) \cong \gamma l \Delta \theta \sin \frac{\theta_{1}+\theta_{2}}{2}$

where $l$ is the perimeter of the triple line. This driving force is balanced by the viscous force $F_{\text {visc }} \cong \eta l v_{\mathrm{cm}}$. Consider that the triple line of floating bubbles is completely depinned. ${ }^{30}$ Assume naively that the contact angle hysteresis $\Delta \theta$ exactly follows the magnetic field; in other words, Equation 2 takes place

2. $\Delta \theta=\widetilde{\theta} \exp \left(-\frac{r_{\mathrm{cm}}}{\lambda}\right) \quad \widetilde{\theta}=\mathrm{const}$

where $r_{\mathrm{cm}}$ denotes the location of the center mass of the bubble. The balance of forces gives rise to the equation (consider that the soap bubbles are treated as zero mass particles)

3. $\alpha \frac{\mathrm{d} r_{\mathrm{cm}}(t)}{\mathrm{d} t}-\beta \exp \left(-\frac{r_{\mathrm{cm}}}{\lambda}\right)=0$

where $\alpha \cong \eta=$ const and $\beta \cong \gamma \widetilde{\theta}=$ const (the numerical factors are omitted for the sake of simplicity). The routine integration of

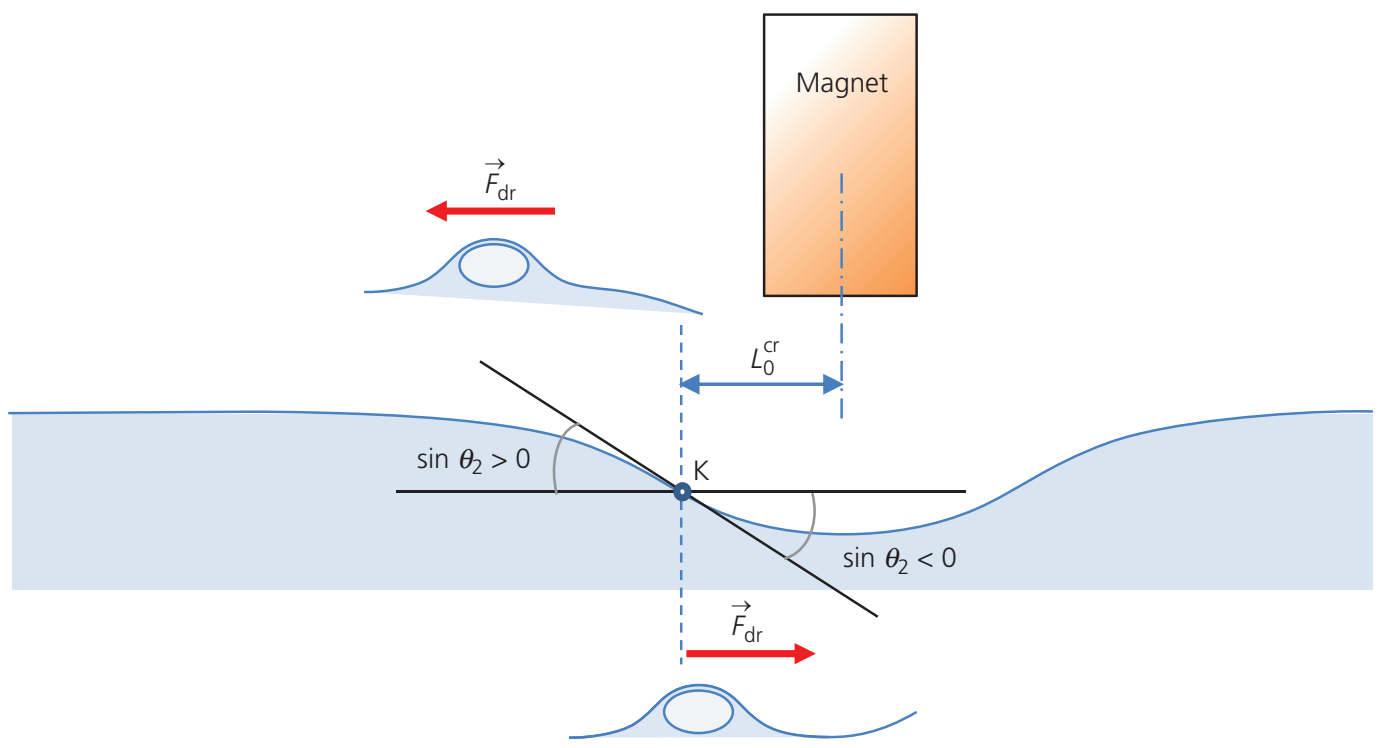

Figure 5. Scheme of the interaction of bubbles with a magnet is shown. The interaction depends on the location of the bubbles on the surface of liquid curved by the magnet. Red arrows indicate the direction of the driving forces $\vec{F}_{\mathrm{dr}}$ 


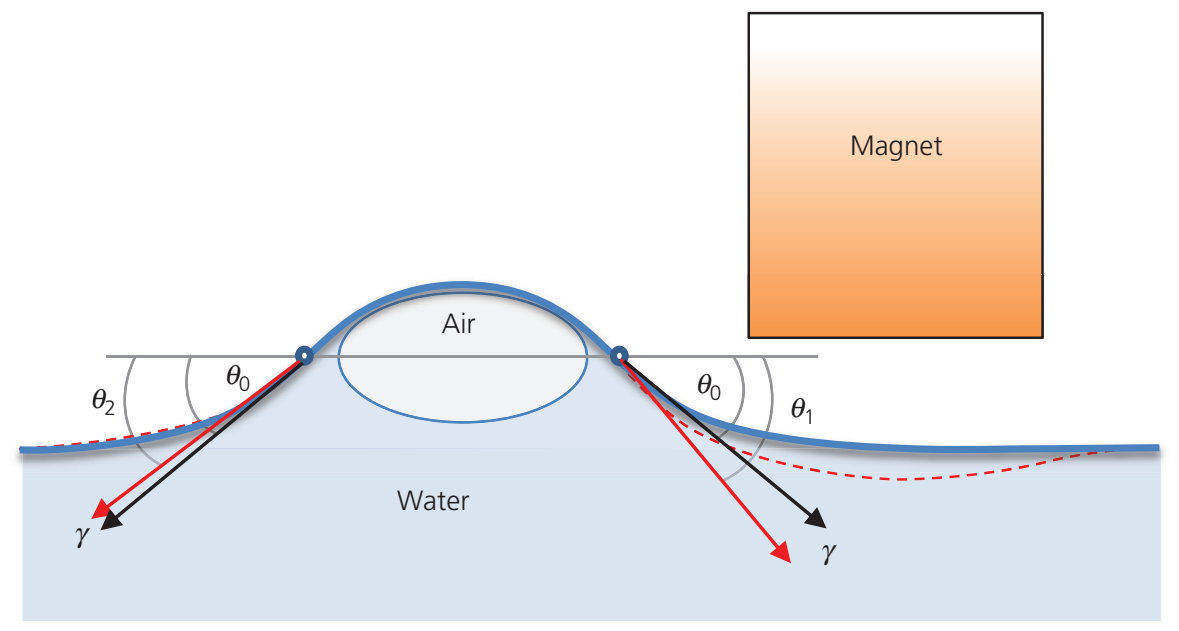

Figure 6. Scheme of the deformation of the soap bubble by the magnetic field is depicted. $\theta_{0}$ denotes the interfacial contact angle of non-deformed liquid/vapor interface; $\theta_{1}$ and $\theta_{2}$ denote the interfacial contact angles of the same surface deformed by the magnet

Equation 3 yields the following scaling law for the velocity of the center mass of the bubbles

4. $v_{\mathrm{cm}}(t) \cong \frac{\lambda}{t}$

Fitting of the experimental data with the power function, shown in Figure 4(b), supplied the best possible approximation given by $v_{\mathrm{cm}}(t) \cong 1.8 \times 10^{-3} / t^{1.014}[\mathrm{~m} / \mathrm{s}]$, which is in more than satisfactory agreement with Equation 4. The numerical value of power (namely, $1.028 \pm 0.01$ ) was obtained by averaging over five experimental curves. It is recognized from Figure 4(b) that the experimental data obtained for various magnet/liquid separations and diameters of the bubbles are well fit by the same master line, thus validating the proposed model, summarized by Equation 3 . Moreover, the value appearing in the numerator of the function fitting the experimental data (namely, $1.8 \times 10^{-3} \mathrm{~m}$ ) is on the order of magnitude of the characteristic length $\lambda$, describing the spatial attenuation of the vertical component of the magnetic field.

\section{Conclusions}

The authors conclude that the proposed qualitative model satisfactorily explains the observed repulsion of soap bubbles by a steady magnetic field, and it is reasonable to attribute the displacement of the bubbles, floating on diamagnetic liquids, to the magnetically inspired deformation of the liquid/vapor interface, known as the Moses effect. It is plausible to suggest that the impact of gravity on the motion of bubbles, seen as zero mass particles, is negligible.

\section{Acknowledgements}

An acknowledgement is made to the donors from the Israel Ministry of Absorption for the partial support of the scientific activity of Dr Mark Frenkel.

\section{REFERENCES}

1. Calcagnile $P$, Fragouli D, Bayer IS et al. (2012) Magnetically driven floating foams for the removal of oil contaminants from water. ACS Nano 6(6): 5413-5419.

2. Lin J, Zhou W, Kumbharb A et al. (2001) Gold-coated iron (Fe@Au) nanoparticles: synthesis, characterization, and magnetic field-induced self-assembly. Journal of Solid State Chemistry 159(1): 26-31.

3. Doyle PS, Bibette J, Bancaud A and Viovy JL (2002) Self-assembled magnetic matrices for DNA separation chips. Science 295(5563): 2237-2238.

4. Whitesides GM and Grzybowski B (2002) Self-assembly at all scales. Science 295(5564): 2418-2421.

5. Vandewalle N, Obara N and Lumay G (2013) Mesoscale structures from magnetocapillary self-assembly. European Physical Journal E 36(10): 127.

6. Lumay G, Obara N, Weyer F and Vandewalle B (2013) Selfassembled magnetocapillary swimmers. Soft Matter 9(8): 2420-2425.

7. den Dulk RC, Schmidt KA, Sabatté G, Liébana $S$ and Prins MWJ (2013) Magneto-capillary valve for integrated purification and enrichment of nucleic acids and proteins. Lab on a Chip 13(1): 106-118.

8. Frenkel M, Danchuk V, Multanen V and Bormashenko E (2018) Magnetic field inspired contact angle hysteresis drives floating polyolefin rafts. Colloid and Interface Science Communications 22(1): 38-41.

9. Frenkel M, Danchuk V, Multanen V et al. (2018) Towards understanding of magnetic displacement of floating diamagnetic bodies, I: experimental findings. Langmuir 34(22): 6388-6395.

10. Tadmor R (2011) Approaches in wetting phenomena. Soft Matter 7(5): 1577-1578.

11. Tadmor R and Yadav PS (2008) As-placed contact angles for sessile droplets. Journal of Colloid and Interface Science 317(1): 241-246.

12. Starov V (1992) Equilibrium and hysteresis contact angles. Advances in Colloid and Interface Science 39(1): 147-173.

13. Eral HB, Mannetje DJCM and Oh JM (2013) Contact angle hysteresis: a review of fundamentals and applications. Colloid and Polymer Science 291(2): 247-260.

14. Hejazi B and Nosonovsky M (2013) Contact angle hysteresis in multiphase systems. Colloid and Polymer Science 291(2): 329-338.

15. Dai Q, Huang W and Wang X (2018) Contact angle hysteresis effect on the thermocapillary migration of liquid droplets. Journal of Colloid and Interface Science 515(1): 32-38. 
16. Chen JZ, Troian SM, Darhuber AA and Wagner S (2005) Effect of contact angle hysteresis on thermocapillary droplet actuation. Journal of Applied Physics 97(1): 014906.

17. Sugawara H, Hirota N, Homma T, Ohta M and Kitazawa K (1996) Magnetic field effect on interface profile between immiscible nonmagnetic liquids - enhanced Moses effect. Journal of Applied Physics 79(8): 4721-4723.

18. Kitazawa K, Ikezoe $Y$, Uetake $\mathrm{H}$ and Hirota N (2001) Magnetic field effects on water, air and powders. Physica B 294-295(1): 709-714.

19. Hirota N, Homma T, Sugawara H et al. (1995) Rise and fall of surface level of water solutions under high magnetic field. Japanese Journal of Applied Physics 34(8A): L991-L993.

20. Haynes WM (ed.) (2010) CRC Handbook of Chemistry and Physics, 91st edn. CRC Press, Boca Raton, FL, USA.

21. Bragg $L$ and Nye JF (1947) A dynamical model of a crystal structure. Proceedings of the Royal Society of London A 190(1023): 474- 481 .

22. Kralchevsky PA and Nagayama K (1994) Capillary forces between colloidal particles. Langmuir 10(1): 23-36.

23. Kralchevsky PA and Nagayama K (2000) Capillary interactions between particles bound to interfaces, liquid films and biomembranes. Advances in Colloid and Interface Science 85(2-3): 145-192.
24. Kralchevsky PA, Paunov VN, Ivanov IB and Nagayama K (1992) Capillary meniscus interactions between colloidal particles attached to a liquid-fluid interface. Journal of Colloid and Interface Science 151(1): 79-92.

25. Wong CYH, Adda-Bedia M and Vella D (2017) Non-wetting drops at liquid interfaces: from liquid marbles to Leidenfrost drops. Soft Matter 13(31): 5250-5260.

26. Rodrıguez-Villarreal Al, Tarn MD, Madden LA et al. (2011) Flow focussing of particles and cells based on their intrinsic properties using a simple diamagnetic repulsion setup. Lab on a Chip 11(7): 1240-1248.

27. Landau L and Lifshitz E (1960) Electrodynamics of Continuous Media: Volume 8 of the Course of Theoretical Physics. Pergamon, Oxford, UK.

28. Zang D, Lin K, Li L et al. (2017) Acoustic levitation of soap bubbles in air: beyond the half-wavelength limit of sound. Applied Physics Letters 110(12): 121602.

29. Furmige CGL (1962) Studies at phase interfaces: I. the sliding of liquid drops on solid surfaces and a theory for spray retention. Journal of Colloid Science 17(4): 309-324.

30. Teixeira MAC, Arscott S, Cox SJ and Teixeira PIC (2015) What is the shape of an air bubble on a liquid surface? Langmuir 31(51): 13708-13717.

\section{How can you contribute?}

To discuss this paper, please submit up to 500 words to the journal office at journals@ice.org.uk. Your contribution will be forwarded to the author(s) for a reply and, if considered appropriate by the editor-in-chief, it will be published as a discussion in a future issue of the journal.

ICE Science journals rely entirely on contributions from the field of materials science and engineering. Information about how to submit your paper online is available at www.icevirtuallibrary.com/page/authors, where you will also find detailed author guidelines. 